\title{
Dispersion in fully developed flow through regular porous structures: Experiments with wire-mesh sensors
}

\author{
Journal Article \\ Author(s): \\ Häfeli, Richard; Hutter, Cédric; Damsohn, Manuel; Prasser, Horst-Michael; Rudolf von Rohr, Philipp \\ Publication date: \\ 2013-07 \\ Permanent link: \\ https://doi.org/10.3929/ethz-a-010121315
}

Rights / license:

Creative Commons Attribution-NonCommercial-NoDerivatives 4.0 International

Originally published in:

Chemical Engineering and Processing 69, https://doi.org/10.1016/j.cep.2013.03.006 


\title{
Dispersion in fully developed flow through regular porous structures: experiments with wire-mesh sensors
}

\author{
R. Häfelia , C. Hutter ${ }^{\mathrm{a}}$, M. Damsohn ${ }^{\mathrm{b}}$, H.-M. Prasser ${ }^{\mathrm{b}}$, Ph. Rudolf von \\ Rohr ${ }^{\mathrm{a}, *}$, \\ ${ }^{a}$ Institute of Process Engineering, ETH Zurich, Sonneggstrasse 3, 8092 Zurich, \\ Switzerland \\ ${ }^{b}$ Institute of Energy Technology, ETH Zurich, Sonneggstrasse 3, 8092 Zurich, Switzerland
}

\begin{abstract}
Within this study the wire-mesh sensor is proposed as a suitable device to investigate radial and axial dispersion in tubular reactors. Axial dispersion in turbulent flow through a regular highly porous structure is addressed and the effect of reactor length on the estimated axial dispersion coefficient is discussed. We state that the gradual increase of turbulence intensity in the entrance section of the porous structure is an effect which leads to a length dependence of the dispersion coefficient. Furthermore, we present measurements of radial mixing by the wire-mesh sensor and compare it to reference measurements using laser induced fluorescence. By that we discuss the possible bias from non-homogeneous tracer distribution between the electrodes. Despite the differences of the measurement principles we found a good qualitative agreement between the results.
\end{abstract}

Keywords: Porous media, Turbulence, Axial dispersion, Length effect, Wire-mesh sensor

\section{Introduction}

The potential to achieve constant product quality with increased safety compared to batch reactors led to a new packed tubular reactor approach which is produced by selective laser sintering [I]. The reactor has an inner

\footnotetext{
*corresponding author. Tel.: +41 4463224 88; Fax: +41 4463213 25; Mail: vonrohr@ipe.mavt.ethz.ch
}

Accepted Author Manuscript. Final publication available at http://dx. doi.org/10.1016/j.cep.2013.03.006 
diameter of $7 \mathrm{~mm}$ and contains a packing with a porosity of $84 \%$. This allows to run chemical reactions with reasonable high flowrates at comparable low pressure drop. A challenge for the design of continuous reactors is the characterization of the residence time distribution (RTD).

The RTD can be measured by tracer pulse experiments. Depending on the application, authors used various different tracers like radioactive substances to measure in pipelines [2] and fluorescent dyes to measure in microreactors [3]. Often used tracer substances are aqueous salt solutions which can be tracked by their conductivity. We decided to use potassium chloride as tracer in water in combination with a wire-mesh sensor for measurement of conductivity.

In this publication we present a new method to characterize the residence time distribution from spatially resolved information. In a further step the technique could be applied to two-phase flow RTD measurements as the wiremesh sensor is well established for measurements in water-air two-phase flow [4]. The experiments performed with a water flow demonstrate the potential of the measurement technique.

With the resulting data the length dependence of the axial dispersion coefficient $D_{L}$ in structured packings is discussed.

\section{Literature review}

The following section attempts to review the most important literature on the length dependence of the dispersion coefficient and on flow in the entrance section of a pipe and of porous structures.

Danckwerts wrote in one of the first publications on the dispersion model: " $D$ should, of course, be independent of $L$ for a given $u$ " [5]. In the same year Taylor showed that in laminar flow through a tube, the axial dispersion can be described by the dispersion model, provided the length of the pipe exceeds a certain minimum [6]. When this condition is not fulfilled the dispersion is non-Fickian and cannot be described by the dispersion model. When the dispersion model is applied to laminar pipe flow without fulfilling this condition, a dispersion coefficient varying with the length is obtained.

Levenspiel and Smith mentioned two conditions for the dispersion model to hold: uniform velocity profile or long enough measurement section [G]. Later studies $([\overline{8}],[\underline{9}])$ on the length effect identified the laminar boundary layer on the surface of a solid as an effect which can not be described by the dispersion model. 
Another important fact to be considered is that the mathematics behind the dispersion model with open-open boundary conditions only lead to a proper residence time distribution if $\frac{D_{L}}{u \cdot L}<0.01$ ([IU], pg. 302). This is primarily because it is unclear what happens at the boundary. It is possible that some of the observed trends of length effects are resulting from a violation of this condition.

The continuing research on this topic shows that no closed theory was developed which allows to exclude all length effects. Delagado mentioned in his review article [II], in a much weaker form than Danckwerts [5], that the dispersion coefficient should be independent of the length, if an experimental method is valid. Nevertheless he then referred to Han et al. [I2], who gave a criterion on the minimum length of a measurement section in order to reach a constant value of dispersion coefficient. A satisfactory explanation why the dispersion coefficient changes with the length is however not given. Han et al. only mentioned that this behaviour is attributed to turbulence effects [12].

In summary it can be stated that the length effect is well known in literature but a conclusive theory is still missing. We will later propose that also the build-up of turbulence (or decay, depending on geometry) in the entrance section of a porous structure leads to a length dependent dispersion coefficient. For that we reflect on some results from literature on developing flow through pipes and porous structures.

In pipes, developing turbulent flow is a transition from a boundary layer type flow at the entrance to a fully developed flow downstream [13]. In turbulent flow the entrance section contains six flow regimes, for which theoretical models were developted [14]. Despite the identification of flow regimes many researchers investigated the length that it takes for the flow to be fully developed. This knowledge is of great importance for the design of rigs for internal flow investigations. Barbin and Jones observed that developed turbulent flow is not attained in a pipe length corresponding to 40 diameters [15]. Weir et al. states that for developed flow it takes longer than usually assumed, in their case more than 70 diameters [16]. Further they observed that the entrance length largely depends on inlet flow conditions. Recent studies present numerical treatments of the entrance flow. Kumara et al. found that for the laminar case after 31.78 diameters the centerline velocity reached $99 \%$ of its final value, what they defined as fully developed [1:3]. In the turbulent case they found that, after an overshoot of centerline velocity at about 30 diameters, developed turbulent flow is attained at about 65 di- 
ameters.

Similar studies in porous structures are rare. While the entrance flow in a pipe is expected to share some characteristics with entrance flow in a porous structure, there are also considerable differences, e.g. the periodic acceleration and deceleration of the flow in porous structures. Mokrani et al. investigated the flow over vortex generators mounted in a pipe [17]. Mean and statistic quantities of the flow were measured using laser Doppler velocimetry. They found that after four rows of vortex generators the flow reaches a steady periodic regime. A porous structure which is better comparable to our structure is investigated by Horneber et al. [18]. By numerical simulations the flow through a row of 8 units of a Kelvin cell is simulated. It is observed that fully developed flow, when judging from the development of mean velocity, is reached after very few cells. Butscher et al. [1.9] used particle image velocimetry with refractive index matching between the solid and the fluid to measure velocity fields inside a porous structure. In the resolved length scales the flow is developed after about two periodic units, what corresponds to 6 cells.

These studies imply that in porous structures the length until the flow is fully developed is much shorter (less than 10 cells) than in pipe flow (more than 50 diameters). The porous structure investigated in our study comprises 87 cells in a length of $0.2 \mathrm{~m}$. We therefore conclude, based on comparison to the above mentioned literature, that the flow is fully developed after less than $0.2 \mathrm{~m}$.

With this publication we contribute to the understanding of axial dispersion by stating that the build-up of turbulence in the entrance section of the porous structure leads to a length dependent dispersion coefficient. We further introduce the wire-mesh sensor as a measurement technique to assess both radial and axial dispersion in tubular reactors.

\section{Theoretical Background}

\subsection{Dispersion model}

Idealized models are used to describe the residence time distribution of a real reactor. We use the dispersion model, which is a one dimensional model for a problem which has concentration and velocity fluctuations in three dimensions. The justification of this model has its origin in the work by Taylor [6]. He showed that even in laminar flow through a tube, where the velocity profile over the cross section is parabolic, the dispersion model is 
applicable provided that the time for convective transport is long compared to the time of decay during which radial variations of concentration are reduced to a fraction of their initial value through the action of molecular diffusion. Later the dispersion model was applied to turbulent flow [20]. By assuming the universal velocity profile over the cross section it was shown that a onedimensional model can describe dispersion in turbulent flow. Today the dispersion model is, along with the tanks in series model, one of the two most important models of dispersion and can be used for turbulent flow in pipes and for flow in packed beds ([10], pg. 293). The dispersion of mass in turbulent flow (stochastic movement of fluid) is similar to diffusion (stochastic movement of molecules). Therefore we use the dispersion model because it describes mass transfer with a coefficient $\left(D_{L}\right)$ which is similar to the diffusion coefficient:

$$
\frac{\partial c}{\partial t}=D_{L} \cdot \frac{\partial^{2} c}{\partial x^{2}}-u \cdot \frac{\partial c}{\partial x}
$$

The mass transfer is composed of convection with velocity $u$ and dispersion with a coefficient $D_{L}$ which contains diffusion and turbulent mixing.

Applying equation (四) to a tubular reactor of length $L$, there are different possible boundary conditions [10]. The open-open boundary condition takes an infinitely long section for calculation, motivated by the fact that there is also dispersion over the entry and the exit of the investigated section. The initial condition for eq. (四) is to have a Dirac pulse at the inlet of a reactor at time zero. As known from literature [6], the solution to eq. (四) for the outlet of the reactor $(x=L)$ leads to the residence time distribution, given by:

$$
E(t)=\frac{1}{\tau \cdot \sqrt{4 \cdot \pi \cdot \frac{D_{L}}{u \cdot L} \cdot t / \tau}} \cdot \exp \left(\frac{-(1-t / \tau)^{2}}{4 \frac{D_{L}}{u \cdot L} \cdot t / \tau}\right)
$$

The dispersion model describes the residence time distribution by only two parameters, i.e. the mean residence time $\tau$ and the dimensionless vessel dispersion number $\frac{D_{L}}{u \cdot L}$. A narrow residence time distribution is associated with a small value of $\frac{D_{L}}{u \cdot L}$.

\subsection{Determination of RTD from pulse experiments}

Injecting a Dirac pulse of tracer at the inlet of the reactor would allow the direct measurement of the residence time distribution at the outlet of 
the reactor. However, if the tracer pulse at the inlet is broad, the outlet signal is given as the convolution of the inlet signal and the residence time distribution:

$$
c_{\text {out }}(t)=\int_{0}^{t} c_{\text {in }}\left(t^{*}\right) \cdot E\left(t-t^{*}\right) \cdot d t^{*}
$$

When the concentration profiles are measured at the inlet and at the outlet, the residence time distribution can be determined by two methods:

A) Deconvolution of the signals by Fourier transformation, filtering and inverse Fourier transformation.

B) Fitting parameters of a RTD model to fulfill equation (国).

We decided to use the second approach, since it allows to treat any experimental data, i.e. with arbitrary $c_{i n}(t)$. The disadvantage is that only residence time distributions described by a model can be determined, whereas the exact RTD remains unknown.

\section{Experimental}

\subsection{Investigated structure}

The packing in the reactor consists of a regular porous structure that is produced by Selective Laser Sintering (SLS). This manufacturing method allows to produce geometries of nearly any shape. Our structure represents the negative pattern of tetrahedrally arranged overlapping spheres (Fig. 四) like first described in the publication by Hutter et al. [21]. The inner diameter of the tubular reactor is $7 \mathrm{~mm}$ and the length is $200 \mathrm{~mm}$. The packed length in this tube is $19.6 \mathrm{~mm}$ and the porosity of this structure is $84 \%$.

\subsection{Measurement technique}

For the measurement of tracer pulses, a wire-mesh sensor was used [4]. The sensor built for this investigation fits in a tubular cross-section with an inner diameter of $7 \mathrm{~mm}$ like shown in Fig. 2 .

It consists of two planes of parallel wires made of stainless steel 1.4304 with a diameter of $50 \mu \mathrm{m}$. The two planes are orthogonal to the flow direction. The distance between two wires of the same plane is $0.78 \mathrm{~mm}$. The distance from the first to the second plane is $0.6 \mathrm{~mm}$. The upstream wires are rotated by 90 degrees against the downstream wires in order to generate a plurality 


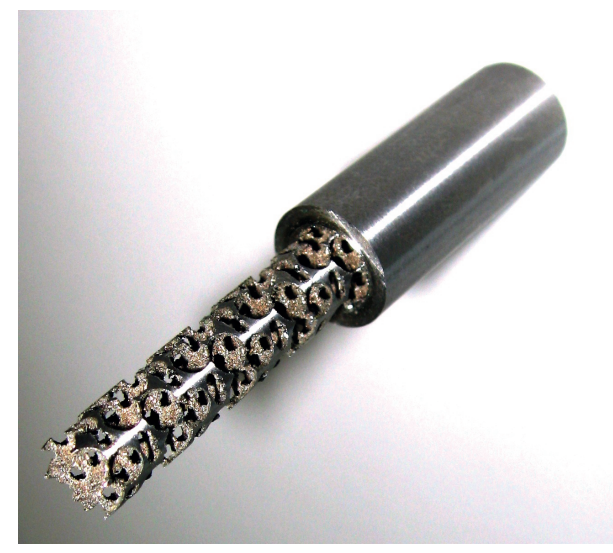

Figure 1: Investigated regular porous structure with partly removed outer tube.

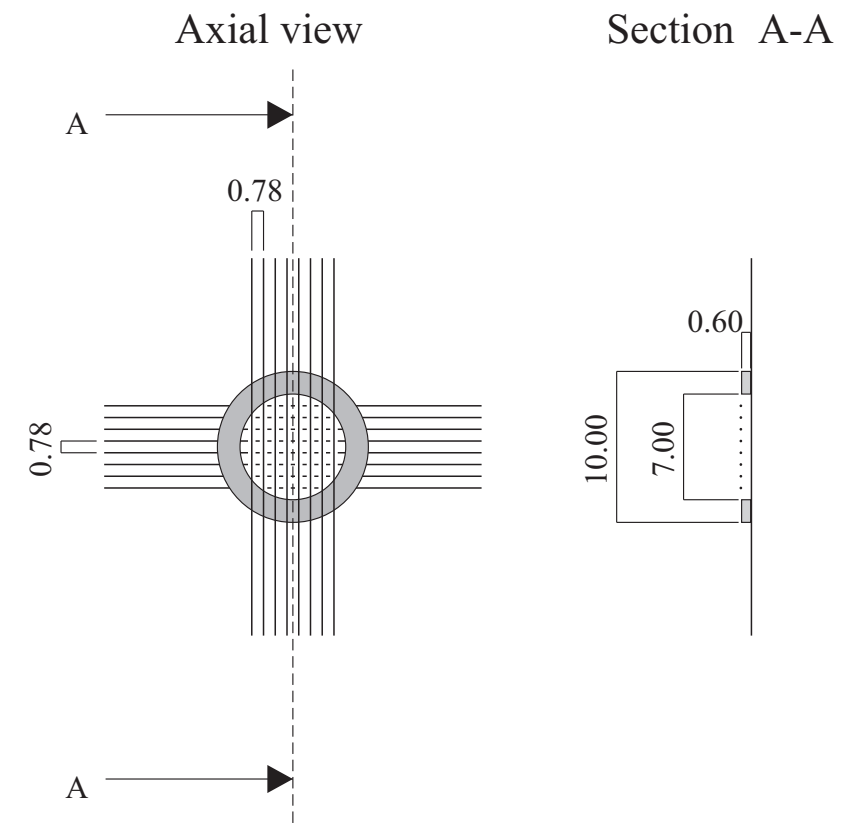

Figure 2: Wire-mesh sensor for measurement of conductivity distribution over cross-section of $7 \mathrm{~mm}$ inner diameter. Dimensions in $\mathrm{mm}$. 
of sensitive nodes. The wires upstream act as transmitters, the wires downstream as receivers. The transmitters are consecutively activated applying a rectangular pulse with a positive and a negative period. The voltage applied to a single transmitter causes a current from the transmitter wire through the measuring volumes to the receiving wires. Operational amplifiers in the transmitter lines guarantee that all non-active wires are kept on ground to avoid any cross talk explained in detail by Prasser et al. [4]. The current arriving at the receiving lines is transformed into a voltage by the operational amplifiers and sampled by individual sample/hold circuits (data aquisition system of teletronic Rossendorf $\mathrm{GmbH}$ ). After an analogue/digital conversion the signals are recorded by a data acquisition computer. The system has a high measurement frequency of $10 \mathrm{kHz}$. The data is finally stored in a matrix whose dimensions are defined by the number of transmitter (number of matrix rows) and receiver wires (number of matrix columns) for every time step. Due to the fact that not all crossing points are within the measurement section we sample only 52 points although we have a sensor with $8 x 8$ wires. The resistance of the wires is very low compared to the resistance of the fluid. The resistance of the wires plus its connections and cables to the AD converter is measured to be $27 \Omega$. The minimum resistance of the fluid in the electrode is $34 k \Omega$ when it has a concentration of $0.3 \mathrm{~g} / \mathrm{l}$. This is the concentration of the tracer pulse which dilutes on the way from injection to the first sensor, leading to a minimum resistance which is higher than $34 k \Omega$ in all experiments. Therefore we can assume that the current at the receiver wires depends linearly on the conductivity of the fluid. In two-phase flow the conductivity is correlated to the liquid volume fraction in the measurement volume (e.g. [22]). In this study the measured signal is correlated to the concentration of a dissolved salt $(\mathrm{KCl})$.

The drawback of the wire-mesh sensor is its intrusivity. However, in our configuration the sensor occupies only $12 \%$ of the cross section by its wires. Compared to the investigated structure, which produces a highly turbulent flow, the sensor intrusivity is assumed to be negligible.

In order to demonstrate that the sensor is able to measure radial concentration profiles a series of radial mixing experiments was performed with the wire-mesh sensor and by laser induced fluorescence (LIF). For the LIF experiments Rhodamine B was injected in front of a packing of $5 \mathrm{~cm}$ length. In a laser sheet at the outlet of the packing the radial concentration distribution was recorded with a camera. For details regarding the employed LIF technique we refer to Hutter et al. [26]. Exactly the same measurements 
were done with the wire-mesh sensor with potassium chloride as tracer substance. The Schmidt numbers $\left(S c=\frac{\nu}{D_{m}}\right)$ of the two substances are 530 for $\mathrm{KCl}$ and 2400 for Rhodamine B. The diffusion coefficient of $\mathrm{KCl}$ was taken as $1.9 \mathrm{e}-5 \mathrm{~cm}^{2} / \mathrm{s}$ according to Harned et al. [2:3]. The diffusion coefficient of Rhodamine B is taken as 4.2e-6 $\mathrm{cm}^{2} / \mathrm{s}$ according to Gendron et al. [24]. The large Schmidt numbers of the two tracers mean that in both experiments the convective transport of species outweighs the diffusive transport at the Reynolds numbers under investigation.

For the evaluation of the mixing performance the coefficient of variation $(\mathrm{CoV})$ is chosen. The $\mathrm{CoV}$ of a single frame is defined as the standard deviation divided by the mean of the concentration distribution over the cross section:

$$
C o V=\frac{\sigma}{c_{\text {mean }}}
$$

The temporal mean $\mathrm{CoV}$ is then calculated as the mean of fifty measurements. A low value represents a homogeneous concentration distribution.

Fig. 3 shows the CoV measured by LIF and with the wire-mesh sensor at different Reynolds numbers. At low flowrates we observe an inhomogeneous tracer distribution. At higher flowrates the mixing performance steadily increases as the flow becomes more turbulent. The $\mathrm{CoV}$ of a well mixed Rhodamine tracer measured by LIF is $7.12 \mathrm{e}-3$. In the experiments we observe a $\mathrm{CoV}$ at the highest flowrate of $8.24 \mathrm{e}-2$. This means we did not reach the limit of the experimental method. The observed flattening of the curve at high flowrates must therefore be physical. What acts against a further decrease is the shorter residence time at higher flowrates.

It has to be mentioned that a quantitative comparison of mixing experiments done with different methods is generally not possible, however trends should be similar allowing a semi-quantitative comparison [25]. There are various reasons like the different temporal and spatial resolution of the two methods. The different spatial resolution, which is $778 \mu \mathrm{m}$ with the wire-mesh sensor and $50 \mu \mathrm{m}$ with LIF, leads to different results for the CoV as length scales smaller than the characteristic sampling size cannot be detected. Further, the used tracers are not ideal because their density is slightly different from the density of the bulk flow. Another reason why the two measurement techniques do not lead to identical results is that the tracers do not have the same diffusion coefficient. And finally the two sensors do not show the same characteristic regarding non-homogeneous tracer within a measurement volume. The local conductivity reading of the wire-mesh sensor is a result of a 


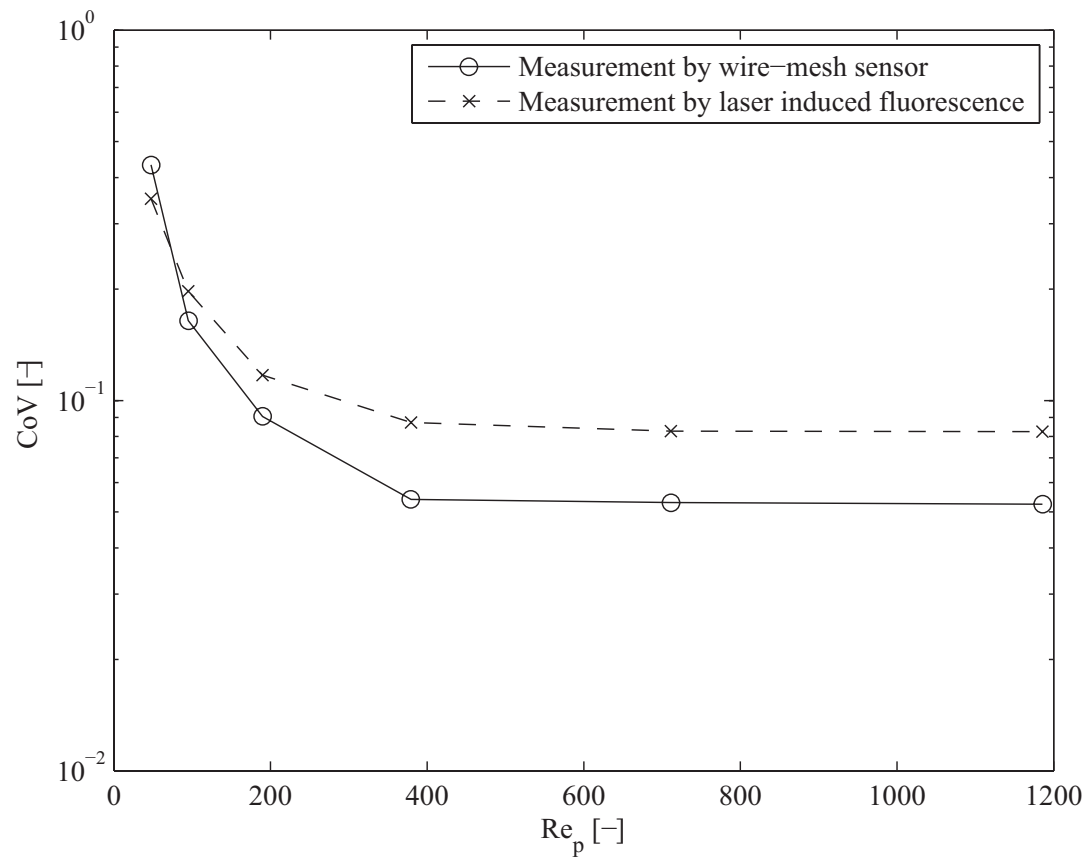

Figure 3: Radial mixing expressed with the coefficient of variation $(\mathrm{CoV})$, measured by LIF and by the wire-mesh sensor. 

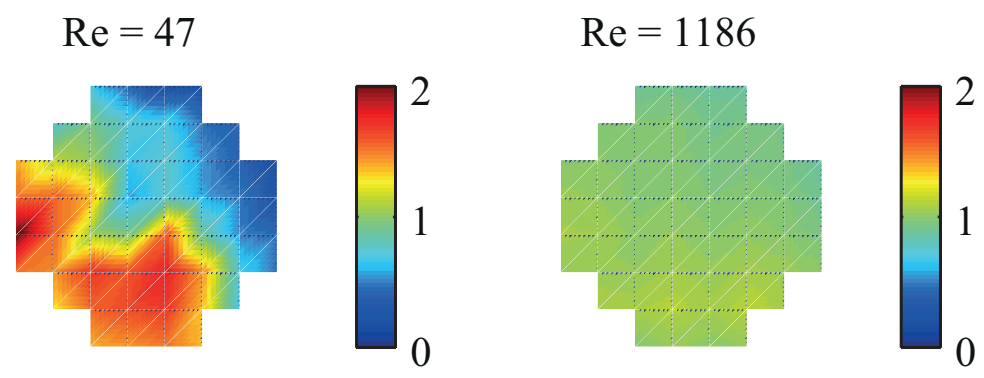

Figure 4: Normalized radial concentration distribution $c / c_{\text {mean }}$ measured by the wire-mesh sensor after a mixing element of $50 \mathrm{~mm}$ length.

three-dimensional electrical potential field established between crossing wires of the sensor. The local conductivity enters as a factor in the potential field equation. In case of strong non-uniformity of the tracer concentration, the sensor signal is therefore not an exact linear average of the tracer concentration within the measuring volume formed by the electrodes. In contrast to that we can expect that the fluorescence intensity in the LIF measurements is always proportional to the mean concentration of Rhodamine in the corresponding volume, irrespective of the spacial distribution of the tracer. This difference might contribute to the deviation between two techniques.

In spite of the differences of the two measurement techniques there is a fairly good agreement of the results (Fig. [1). The maximal deviation of the $\mathrm{CoV}$ obtained by the wire-mesh sensor to the one obtained by LIF is $36.3 \%$. For both measurement techniques the $\mathrm{CoV}$ is decreasing with increasing Reynolds number and the absolute values are also comparable.

Fig. W shows a normalized concentration distribution over the cross section measured by the wire-mesh sensor. It is observed that at low flowrates $\left(R e_{p}=47\right)$, the tracer is inhomogeneously spread over the cross section, i.e. it is concentrated on the bottom left of the reactor. At higher flowrates the turbulent mixing increases, leading to a smooth tracer distribution over the cross-section.

\subsection{Experimental setup}

The experimental setup for the axial dispersion measurements is shown in Fig. 1 with a detail drawing of the arrangement at the inlet wire-mesh sensor. The configuration at the outlet sensor is similar. The demineralized 


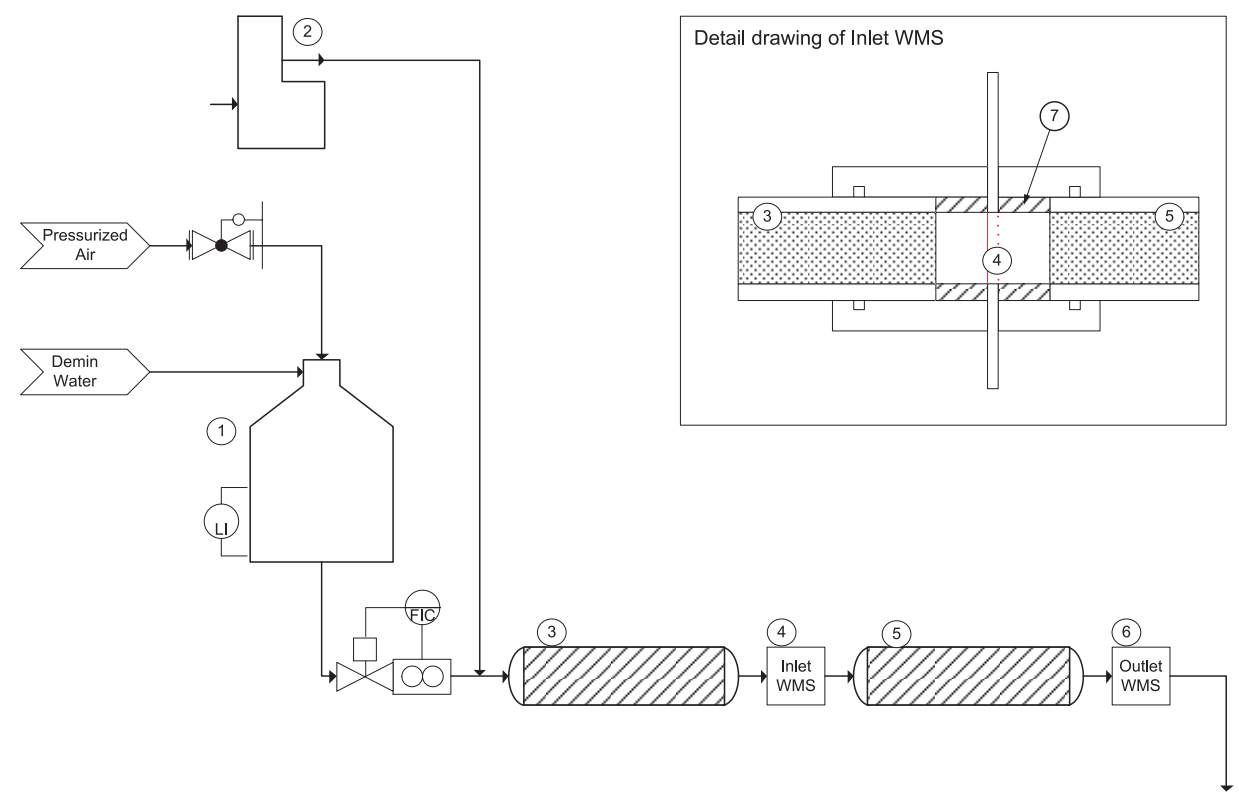

Figure 5: Experimental setup used for pulse experiments. 1: demineralized water tank pressurized by an air cushion up to 7bar. 2: Syringe pump for tracer pulse injection. 3: First reactor (premixing section). 4: inlet wire-mesh sensor. 5: Second reactor (measurement section). 6: Outlet wire-mesh sensor. 7. Teflon ring for defined axial spacing of $5 \mathrm{~mm}$. 
water is supplied from a tank pressurized with air. The mass flow controller (Bronkhorst CORI-FLOW M55C4) allows to control the flow up to $180 \mathrm{~kg} / \mathrm{h}$ with an accuracy of $0.2 \%$. The water flows through a calming section of $1 \mathrm{~m}$ length and $7 \mathrm{~mm}$ diameter before it enters the first reactor. The function of the first reactor (Fig. 5) is to homogenize the tracer in radial direction and to allow the flow field to develop. The dispersion is then measured over the second reactor where, based on the literature reviewed above, we expect fully developed flow. The distance of the wire-mesh to the porous structure is $5 \mathrm{~mm}$ in axial direction to avoid electrical current through the porous structure. For the pulse injection a syringe pump was used with $\mathrm{KCl}$ as tracer substance.

\subsection{Signal postprocessing}

Due to manufacturing tolerance not every node of the wire-mesh sensor has the same sensitivity. For this reason a calibration measurement has to be done. This is achieved by measuring the conductivity of water with a well defined high salt concentration and of demineralized water. The measured signal from the experiment can then be linearly interpolated between the concentrations in the calibration. When the tracer is not distributed homogeneously it is questionable wheter we can assume a linear characteristic between conductivity and concentration. We consider this as an error source in the measurements of radial mixing discussed above. However in the measurements of axial dispersion we will show that the tracer distribution is one-dimesional (Fig. ש). This justifies the assumption of proportionality between tracer concentration and conductivity.

For the evaluation of the tracer pulse experiments the mean value over the cross section of both inlet and outlet sensor was calculated. Further, when no tracer mass is lost in the reactor, the inlet and outlet mass flow has to be equal. Therefore, the area under the curves $c_{i n}(t)$ and $c_{\text {out }}(t)$ were both normalized to be 1 . To these two signals the deconvolution procedure described in 3.2 is applied, i.e. the mean residence time $\tau$ and vessel dispersion number $\frac{D_{L}}{u \cdot L}$ are fitted to fulfill eq. 3 .

\section{Results and Discussion}

\subsection{Mean residence time}

The normalized concentration measured at the single nodes of the wiremesh sensor during a pulse experiment are depicted in Fig. G. Only minor 


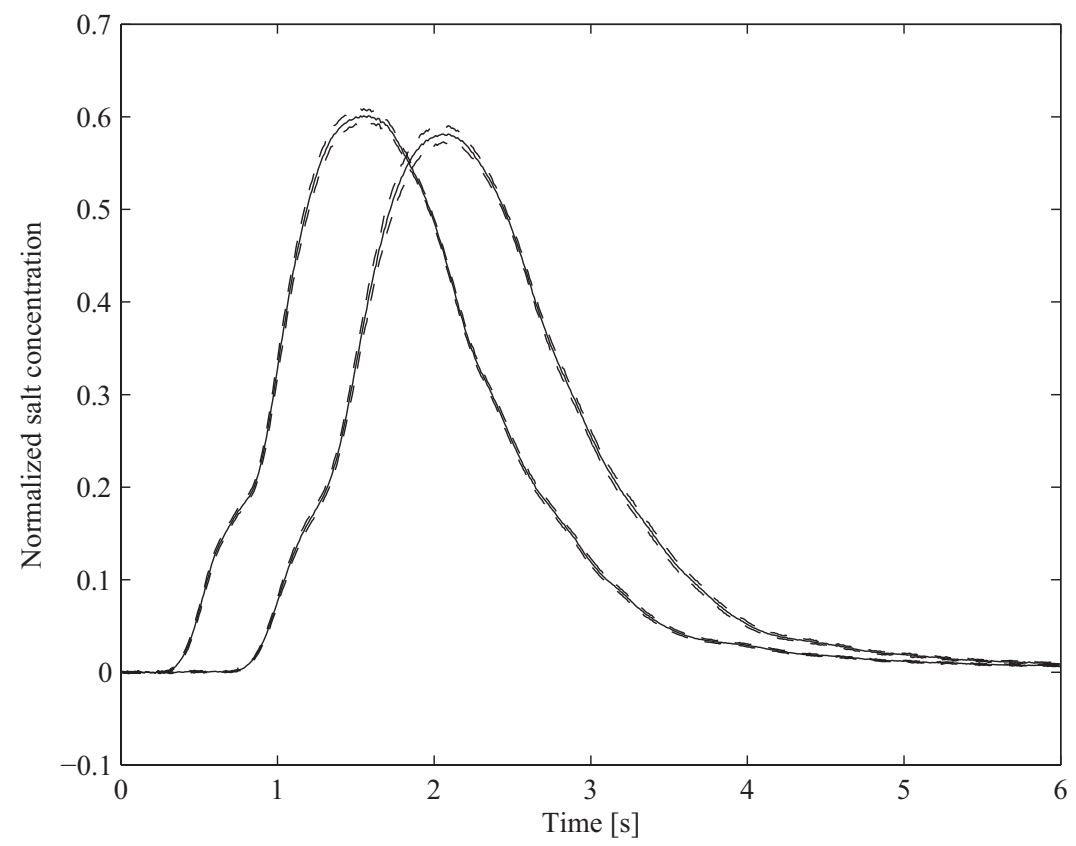

Figure 6: Tracer pulse at inlet and outlet sensor. Mean value (solid) and standard deviation (dashed) of 52 nodes in a cross-section. 
concentration variations are observed at individual nodes within the measurement plane, i.e. no radial concentration gradient of tracer is detected. For a better visualisation of the tracer peak Fig. $\square$ shows the measurements at the nodes of one wire near the center of the tube as a pseudo sideview. This graph shows the quality of the radial mixing of the tracer. Most importantly the tracer arrives as a plug, i.e. simultaneously at the center and at the boundary of the tube. We conclude that the radial mixing is very fast. This implies that the axial dispersion is not controlled by velocity gradients like known from laminar pipe flows. It is clear that with other electrodes this information cannot be measured, what emphasizes the advantages of the wire-mesh sensor for the measurement of dispersion.

The measured mean residence time can be compared to the expected one, defined as:

$$
\tau_{\text {exp }}=\frac{L}{u}=\frac{L}{\dot{V} /\left(d^{2} \cdot \frac{\pi}{4} \cdot \varepsilon\right)}
$$

with the length of the reactor $L$, inner diameter $d$, volume flow $\dot{V}$ and the porosity of the inner structure $\varepsilon$. This is compared to the mean residence time estimated from the pulse experiments. The comparison in Fig. 8 shows a good agreement. The deviation is increasing with increasing residence time, which is attributed to the higher uncertainty of the flow controller at low flowrates. The relative deviation is always lower than $9 \%$.

\subsection{Dispersion coefficient}

The pore Reynolds number $R e_{p}=\frac{u \cdot d_{p}}{\nu}$ is often used for a non dimensional description of the flowrate. This is motivated by the assumption that the characteristic length scale for the flow through the porous structure is the diameter of the pores. It is defined to be the diameter of the opening between the cell like in [26], in our case that is $0.92 \mathrm{~mm}$. In Fig. Q 9 the dispersion coefficient is plotted over the pore Reynolds number. Each point reflects the mean of five pulse experiments. The standard deviation of these five experiments is very low for all Reynolds numbers except at $R e_{p}=380$ where the standard deviation is $21 \%$. The reason is that one of the five experiments is an outlier which led to the high standard deviation. With increasing Reynolds number the dispersion coefficient increases, what is explained by the enhanced turbulence of the flow.

Fig. [1] shows a comparison to the measurements of Hutter et al. [T]. They investigated two unregular foam structures with 20ppi and 30ppi (pores 

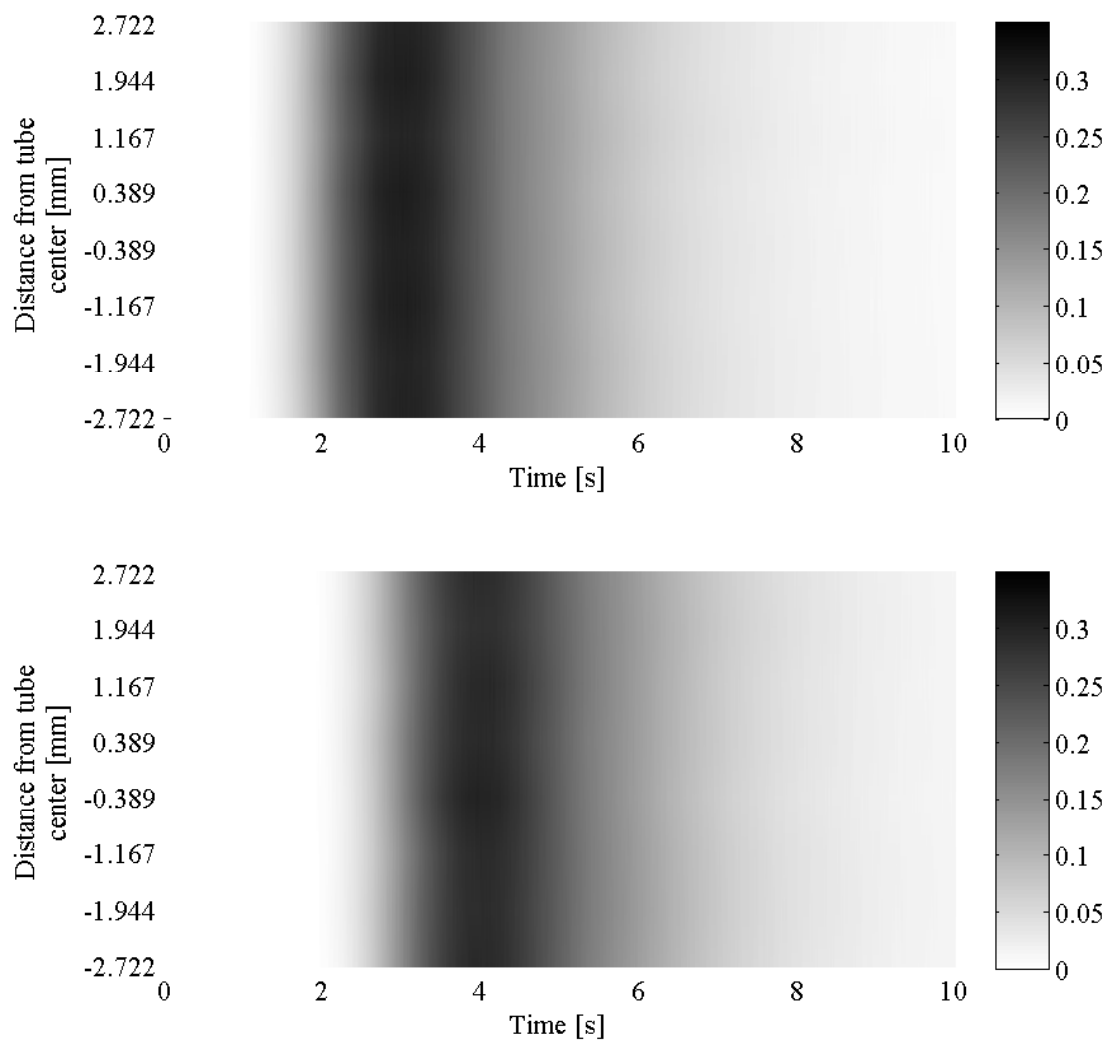

Figure 7: Pseudo side-view of tracer pulse (normalized concentration $\frac{c(t)}{\int_{0}^{\infty} c(t) d t}$ ) at inlet (top) and outlet (bottom). The graph contains the measurements of a wire in the center of the tube at pore Reynolds number $R e_{p}=190$. This graph shows that a radial concentration gradient was not observed in the measurement of axial dispersion. 


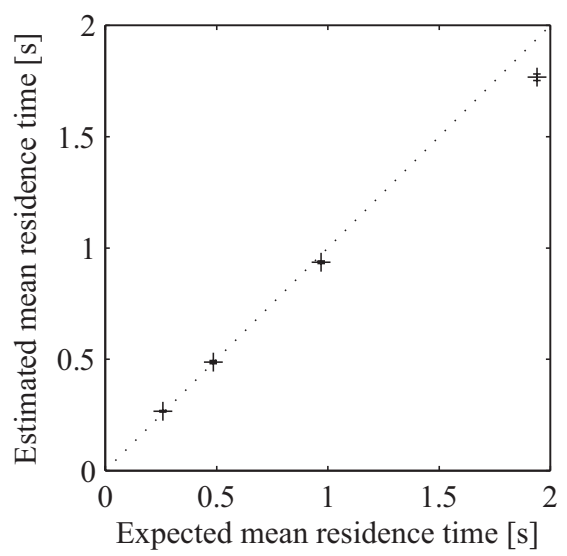

Figure 8: Estimated mean residence time versus expected mean residence time.

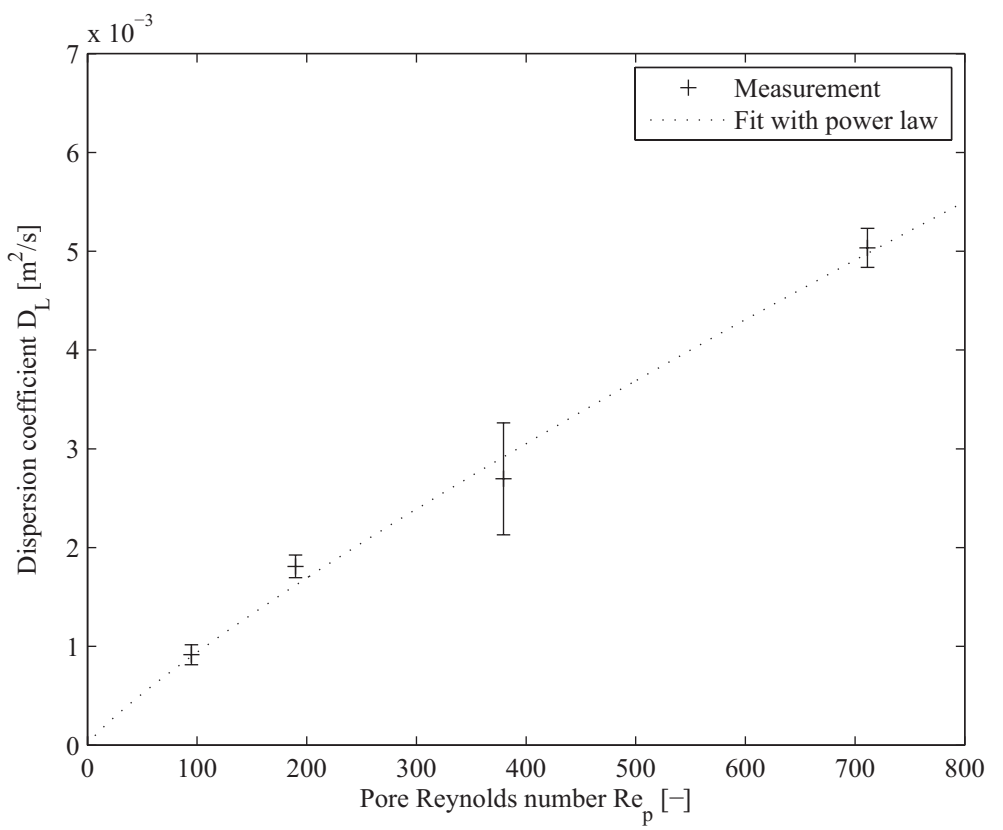

Figure 9: Dispersion coefficient for different pore Reynolds numbers. 


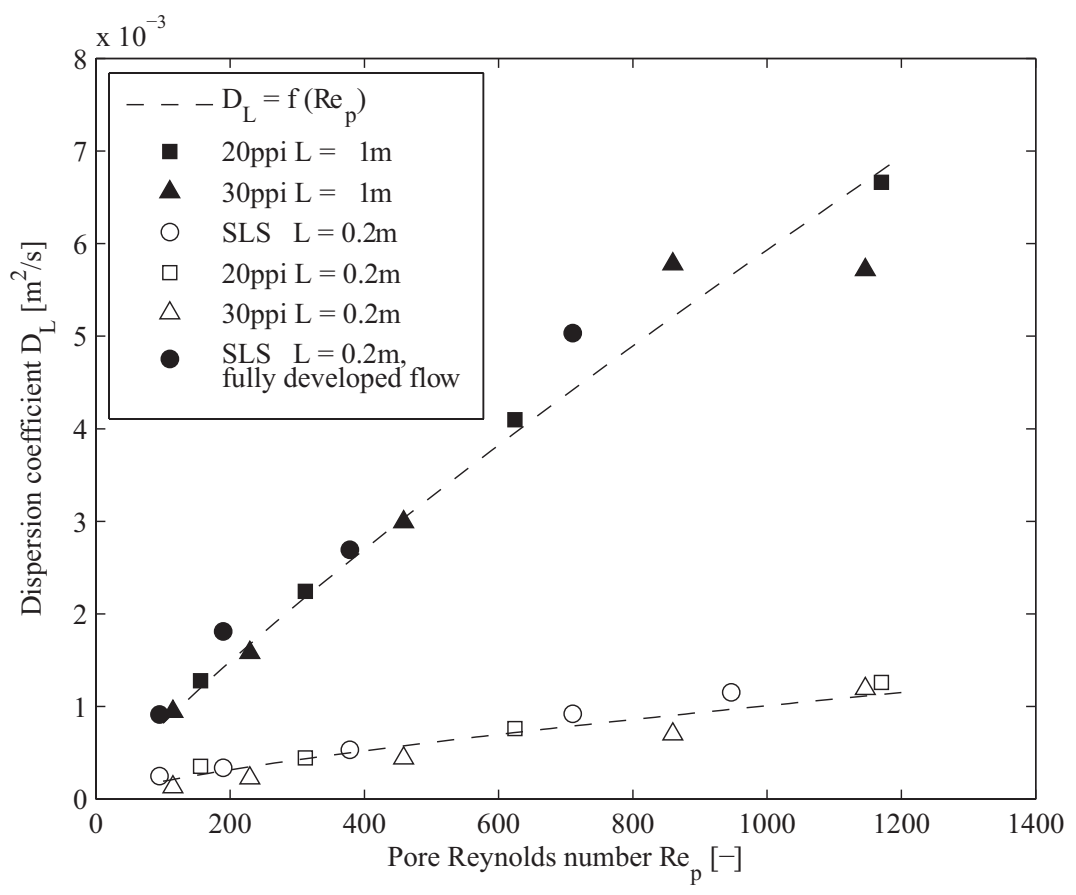

Figure 10: Dispersion coefficients in fully developed flow through the selective laser sintered (SLS) structure of $0.2 \mathrm{~m}$ length, compared to experiments by Hutter et al. [I]. Experiments where the entrance section plays a major role are represented by white symbols. 


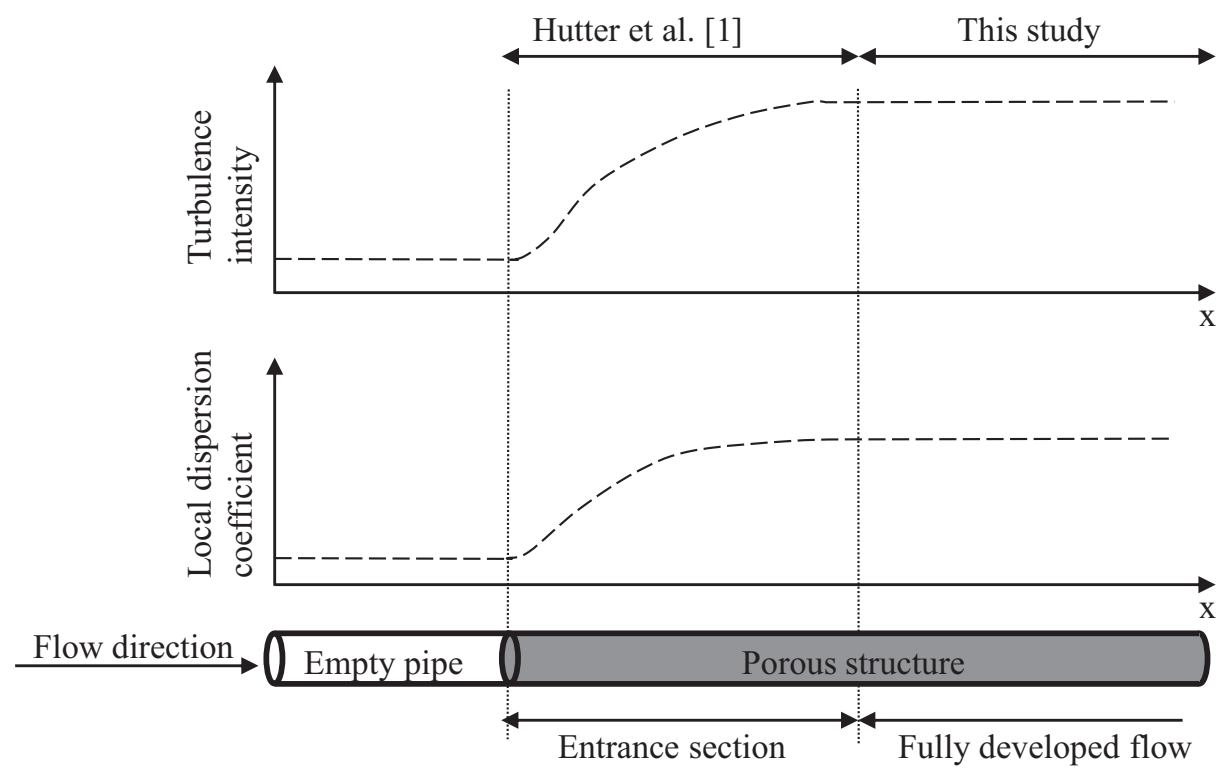

Figure 11: Build-up of turbulence intensity and local dispersion coefficient in the entrance section of a porous structure (qualitative illustration).

per inch) and compared it to the same streamwise periodic structure like the one investigated in this work. They observed that the different packings of $0.2 \mathrm{~m}$ length have similar axial dispersion coefficients (white symbols in Fig (101). Investigating the same structures of $1 \mathrm{~m}$ length, they observed a higher dispersion coefficient $(\boldsymbol{\square}, \mathbf{\Lambda})$. In our study, we measured in fully developed flow through a structured foam of $0.2 \mathrm{~m}$ length $(\bullet)$. The axial dispersion coefficient in this setup was more than four times higher than in the entrance section (o) even though the length of the measurement section was equal.

The gradual build-up of turbulence intensity in the entrance section of the structure is an effect which leads to a dependence of the dispersion coefficient on the position in the reactor. Fig. $\square$ illustrates the evolution of turbulence intensity when an empty pipe flow enters a porous structure. Because the dispersion coefficient also reflects mass transport by turbulence, the local dispersion coefficient illustrated in Fig. $\square$ changes gradually with the buildup of turbulence. When a measurement is made in a section of length $l$ from $x$ to $x+l$, an integral dispersion coefficient characteristic for the section from $x$ to $x+l$ is measured. When measuring in the entrance section, the (integral) 
dispersion coefficient is lower than in a section further downstream. With longer measurement section the entrance section becomes negligible and a constant dispersion coefficient is obtained.

Using a premixer of $0.2 \mathrm{~m}$ length before the actual measurement section, we ensure to measure the dispersion coefficient in fully developed flow. This is supported by the good agreement of the data measured in this study with the ones of Hutter et al. [1].

\section{Conclusions}

Wire-mesh sensors were used for pulse experiments in a regular highly porous structure. Measurements were made at different positions but with equal length of the measurement section. This showed that in the entrance section the dispersion coefficient is lower than further downstream. We discussed the entrance flow of a fluid into a porous structure. The build-up of the radial velocity profile and of the turbulence intensity is introduced as an effect which produces a length dependence of the dispersion coefficient. In our case the flow in the empty tube is laminar and changes to a turbulent flow when it enters the porous structure. The dispersion coefficient is therefore expected to be lower at the entrance than in fully developed flow.

In the face of these results it was shown that the wire-mesh sensor is a suitable technique to characterize radial and axial mass transfer in situ with a high temporal resolution. 


\section{Nomenclature}

Roman symbols

C

d

$\mathrm{d}_{p}$

$\mathrm{D}_{L}$

$\mathrm{D}_{m}$

$\mathrm{E}(\mathrm{t})$

$\mathrm{L}$

$\operatorname{Re}_{p}$

$\mathrm{Sc}$

$\mathrm{t}$

u

$\dot{V}$

$\mathrm{X}$

Greek letters

$\varepsilon$

$\nu$

$\sigma$

$\tau$

Concentration of a tracer substance $[\mathrm{mol} / \mathrm{l}]$

Inner diameter of tubular reactor $[\mathrm{m}]$

Pore diameter $[\mathrm{m}]$

Dispersion coefficient in axial (longitudinal) direction $\left[\mathrm{m}^{2} / \mathrm{s}\right]$

Molecular diffusion coefficient $\left[\mathrm{m}^{2} / \mathrm{s}\right]$

Residence time distribution function $[1 / \mathrm{s}]$

Length of measurement section $[\mathrm{m}]$

Pore Reynolds number [-]

Schmidt number [-]

Time [s]

Interstitial velocity $[\mathrm{m} / \mathrm{s}]$

Volumetric flowrate $\left[\mathrm{m}^{3} / \mathrm{s}\right]$

Axial coordinate $[\mathrm{m}]$

Porosity of porous structure [-]

Kinematic viscosity $\left[\mathrm{m}^{2} / \mathrm{s}\right]$

Standard deviation

Mean residence time $[\mathrm{s}]$

\section{Abbreviations}

$\mathrm{CoV}$

Coefficient of variation

LIF

Laser induced fluorescence

PDE

ppi

Partial differential equation

RTD

Pores per inch

Residence time distribution

SLS

WMS

Selective laser sintering

Wire-mesh sensor 
Sub- and superscripts

$\begin{array}{ll}\text { exp } & \text { Experimental } \\ \text { in } & \text { Limit of measurement section in upstream direction } \\ \text { mean } & \text { Averaged quantity } \\ \text { out } & \text { Limit of measurement section in downstream direction } \\ \text { reac } & \text { Reaction }\end{array}$

\section{Acknowledgements}

We gratefully acknowledge financial support from the Swiss Confederation's innovation promotion agency (CTI) in cooperation with DSM Nutritional Products and Premex Reactor AG.

\section{References}

[1] Hutter C., Zenklusen A., Lang R., Rudolf von Rohr Ph., Axial dispersion in metal foams and streamwise-periodic porous media. Chem. Eng. Sci., 66 (2011), $1132-1141$.

[2] Hull D. E., Kent J. W., Radioactive tracers to mark interfaces and measure intermixing in pipelines. Ind. Eng. Chem., 44(11) (1953), 27452750 .

[3] Trachsel F., Günther A., Khan S., Jensen K.F., Measurement of residence time distribution in microfluidic systems. Chem. Eng. Sci., 60 (2005), $5729-5737$.

[4] Prasser H.-M., Böttger A., Zschau J., A new electrode-mesh tomograph for gas-liquid flows. Flow Meas. Instrum., 9 (1998), 111 - 119.

[5] Danckwerts P.V., Continuous flow systems. Chem. Eng. Sci., 2 (1953), $1-13$.

[6] Taylor G., Dispersion of soluble matter in solvent flowing slowly through a tube. Proc. Roy. Soc. (Lond.), A219(168) (1953), $186-203$.

[7] Levenspiel O., Smith W.K., Notes on the diffusion-type model for the longitudinal mixing of fluids in flow. Chem. Eng. Sci., 6 (1957), 227-235. 
[8] Koch D. L., Brady J. F., Dispersion in fixed beds. J. Fluid. Mech., 154 (1985), $399-427$.

[9] Koch D. L., Brady J. F., Nonlocal dispersion in porous media: nonmechanical effects. Chem. Eng. Sci., 42 (1987), 1377 - 1392.

[10] Levenspiel O., Chemical Reaction Engineering. 3rd ed. John Wiley and Sons, 1999.

[11] Delgado, A critical review of dispersion in packed beds. Heat Mass Transf., 42 (2006), $279-310$.

[12] Han N.-W., Bhakta J., Carbonell R.G., Longitudinal and lateral dispersion in packed beds: effect of column length and particle size distribution. AlChe J., $31-2$ (1985), $277-288$.

[13] Kumara W.A.S., Halvorsen B.M., Melaaen M.C., Computational study on non-asymptotic behaviour of developing turbulent pipe flow. $A d$ vances in Fluid Mechanics VIII., 69 (2010), 39 - 52.

[14] Salami L.A., An investigation of turbulent developing flow at the entrance to a smooth pipe. Int. J. Heat and Fluid Flow, 7(4) (1986), $247-257$.

[15] Barbin A.R., Jones J.B., Turbulent flow in the inlet region of a smooth pipe. J. of Basic Engineering, 85(1) (1963), $29-34$.

[16] Weir J., Priest A.J., Sharan V.K., The effect of inlet disturbances on turbulent pipe flow. J. Mech. Eng. Sci., 16(3) (1974), $211-213$.

[17] Mokrani A., Castelain C., Peerhossaini H., Experimental study of the influence of the rows of vortex generators on turbulence structure in a tube. Chem. Eng. Proc., 48 (2009), 659 - 671.

[18] Horneber T., Rauh C., Delgado A., Fluid dynamic characterisation of porous solids in catalytic fixed-bed reactors. Micropor. Mesopor. Mater., 154 (2012), $170-174$.

[19] Butscher D., Hutter C., Kuhn S., Rudolf von Rohr Ph., Particle image velocimetry in a foam-like porous structure using refractive index matching: a method to characterize the hydraulic performance of porous structures. Exp. Fluids, 43 (2012), 1123 - 1132. 
[20] Taylor G., The dispersion of matter in turbulent flow through a pipe. Proc. Roy. Soc. (Lond.), A223(1155) (1958), $446-468$.

[21] Hutter C., Zenklusen A., Kuhn S., Rudolf von Rohr Ph., Large eddy simulation of flow through a streamwise-periodic structure. Chem. Eng. Sci., 66 (2011), $519-529$.

[22] Prasser H.-M., Misawa M., Tiseanu I., Comparison between wire-mesh sensor and ultra-fast X-ray tomograph for an air-water flow in a vertical pipe. Flow Meas. Instrum., 16 (2005), $73-83$.

[23] Harned H., Nuttall R. L., The diffusion coefficient of potassium chloride in dilute aqueous solutions. J American Chem Soc, 69 (1947), 736 - 740.

[24] Gendron P.-O., Avaltroni F., Wilkinson K.J., Diffusion coefficients of several rhodamine derivatives as determined by pulsed field gradientnuclear magnetic resonance and fluorescence correlation spectroscopy. $J$ Fluoresc., 18 (2008), 1093 - 1101.

[25] Wadley R., Dawson M.K., LIF measurements of blending in static mixers in the turbulent and transitional flow regimes. Chem. Eng. Sci., 60 (2005), $2469-2478$.

[26] Hutter C., Allemann C., Kuhn S., Rudolf von Rohr Ph., Scalar transport in a milli-scale metal foam reactor. Chem. Eng. Sci., 65 (2010), $3169-$ 3178. 